\title{
Estrategia del servicio a domicilio y permanencia de negocios en tiempos de COVID-19.
}

\author{
DOI: https://doi.org/10.33262/ap.v3i3.65
}

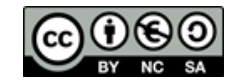

Home service strategy and business permanence in times of COVID-19

\begin{abstract}
Miguel Alejandro Estéfano Almeida. ${ }^{1}$, Estefanía Beatriz Llerena Erazo. ${ }^{2}$, Patricia Belén Morales González. ${ }^{3}$ \& Jonathan Francisco Quiroz Moncerrad. ${ }^{4}$.
\end{abstract}

\begin{abstract}
.
The COVID - 19 pandemic generated a global and national economic crisis, which has hit commercial establishments in the microenterprise sector to a great extent. Therefore, the general objective was to establish the importance of home service in the permanence of businesses in times of the COVID-19 pandemic, due to the fact that the strategy of many owners of commercial and service premises had to visit the customer at home, to sell their goods and services. The descriptive, quantitative methodology was applied, formulating surveys to 384 commercial establishments in the south of Guayaquil, whose results showed a 50\% reduction in sales in most of these stores, with the confinement of 2020 , also observing a recovery of $20 \%$ of their income, in the quarantine of 2021, thanks to the home service strategy. In conclusion, a direct correlation was obtained (Pearson's coefficient greater than 0.9 and p-value less than 0.05 ), between the application of the home service strategy and the increase in income in the aforementioned commercial establishments, during confinements.

\footnotetext{
${ }^{1}$ Estefano Almeida Miguel Alejandro, Institución: Instituto Tecnológico Superior Vicente Rocafuerte, Guayaquil, Ecuador, email: mestefano@istvr.edu.ec, https://orcid.org/0000-0003-4246-8958

${ }^{2}$ Llerena Erazo Estefanía Beatriz, Institución: Instituto Tecnológico Superior Vicente Rocafuerte, Guayaquil, Ecuador, email: ellerena@istvr.edu.ec, https://orcid.org/0000-0003-3845-3673

${ }^{3}$ Morales González Patricia Belén, Institución: Unidad Educativa Fiscal Camilo Gallegos Domínguez, Guayaquil, Ecuador, email: patriciab.morales@educacion.gob.ec, https://orcid.org/0000-0001-88637484

${ }^{4}$ Quiroz Moncerrad Jonathan Francisco, Institución: Instituto Tecnológico Superior Vicente Rocafuerte, Guayaquil, Ecuador, email: jquiroz@ istvr.edu.ec, https://orcid.org/0000-0003-3009-9972
} 
Keywords: Strategy, home service, sales revenue, lockdowns

\section{Resumen.}

La pandemia del COVID - 19 generó una crisis económica mundial y nacional, que ha golpeado en gran medida a los establecimientos comerciales del sector microempresarial. Por ello, se planteó como objetivo general, establecer la importancia del servicio a domicilio en la permanencia de los negocios en tiempos de la pandemia COVID - 19, debido a que la estrategia de muchos dueños de locales comerciales y de servicios, tuvieron que visitar al cliente en su domicilio, para vender sus bienes y servicios. Se aplicó la metodología descriptiva, cuantitativa, formulando encuestas a 384 establecimientos comerciales del sur de Guayaquil, cuyos resultados evidenciaron una reducción del 50\% de las ventas en la mayoría de estos locales, con el confinamiento del 2020, observándose también, una recuperación del $20 \%$ de sus ingresos, en la cuarentena del 2021, gracias a la estrategia del servicio a domicilio. En conclusión, se obtuvo una correlación directa (coeficiente de Pearson superior a 0,9 y p-valor menor a 0,05), entre la aplicación de la estrategia del servicio a domicilio y el incremento de los ingresos en los establecimientos comerciales en mención, durante los confinamientos.

Palabras claves: Estrategia, servicio a domicilio, ingresos por ventas, confinamientos.

\section{Introducción}

La pandemia del COVID - 19 ha generado que los gobiernos en el mundo entero, tomen medidas de confinamiento de la población y cierre de los establecimientos comerciales para evitar aglomeraciones, situación que causó pérdidas por 100,8 millones de empleos en el mercado mundial (Del Pino y Li Gayoso, 2020), afectando severamente al sector económico conformado por los microempresarios. Efectivamente, Canseco et al. (2020), consideran que "las cuarentenas han generado el cierre parcial de restaurantes, establecimientos de expendio de comidas rápidas, panaderías, tiendas", mientras que otros sectores como "el transporte público, hostales, entre otros, atienden del 30\% al 50\% de su capacidad" (p. 64).

Pero no sólo los decretos gubernamentales que han dictaminado los confinamientos de la población, están relacionados con el problema que atraviesa el sector microempresarial, sino también la actitud de la propia población, cuyo comportamiento ha cambiado desde que fueron informados de los peligros del contagio del COVID - 19, comprando a distancia (Coronel et al., 2020), sobre todo por el riesgo de mortalidad, generando cambios en el comportamiento del consumidor, con menor riesgo al endeudamiento, reducción de la compra de cosas consideradas no indispensables y mayor abastecimiento de alimentos (Casco, 2020). Es claro que la pandemia ha frenado el crecimiento de la oferta, a causa del debilitamiento de los hábitos de consumo en la demanda (Blackman et al., 2020). 
De esta manera, la microempresa es la gran afectada por las restricciones de la movilidad durante el tiempo que la pandemia del COVID - 19 (Palomino y Palomino, 2021), se ha mantenido activa en Latinoamérica y en el mundo entero, pero perjudicando en gran medida a la economía latinoamericana, en donde de 5 a 7 MIPYMES, aportan más del $50 \%$ del PIB de las naciones de esta región (Giles, 2020). En México, por ejemplo, hubo una reducción del 35\% de comercios operando en el 2020, con respecto al 2019 (Montejo et al., 2020).

Uno de los efectos de la pandemia COVID - 19 en el sector microempresarial nacional, se vio reflejada en la reducción del precio de venta de algunos de los productos de primera necesidad, que se expenden en mercados y tiendas de abastos del país, e incluso en restaurantes, de acuerdo a las cifras del Instituto Nacional de Estadísticas y Censos, que reflejaron una diminución del Índice de Precios al Consumidor (IPC) acumulado de enero a diciembre, en 0,30\% en el año 2020 (INEC, 2020). Este resultado, fue un impacto económico negativo, derivado de los efectos que generó la infección por coronavirus, desde su llegada al Ecuador (Barahona, 2021).

A estos resultados se añade que en la ciudad de Manta, durante el primer confinamiento expedido el 12 de marzo del 2020, bajo el Acuerdo Ministerial No. 00126-2020, mediante el cual, el (Ministerio de Salud Pública, 2020) dictaminó la Emergencia Sanitaria en todo el territorio nacional, la cual fue reafirmada en el Decreto Ejecutivo No. 1052 (Presidencia de la República del Ecuador, 2020) y el Decreto Ejecutivo No. 1017 (Presidencia de la República del Ecuador, 2020), por lo menos el 80\% de los pequeños negocios no trabajó, debido a la crisis sanitaria, y, fueron muy pocos los que laboraron bajo la modalidad del servicio a domicilio (Castro, 2020).

Sin embargo, a pesar de la problemática del confinamiento y reducción de las compras por parte del consumidor, algunos estudios realizados en Latinoamérica, señalan la gran importancia de la entrega a domicilio de los clientes, en tiempos de la pandemia del COVID - 19. Así por ejemplo, un artículo desarrollado en México, Brasil, Colombia y Ecuador, que seleccionó una muestra de 2.565 consumidores, a través de una encuesta online, reveló que los usuarios se han adaptado a las compras a domicilio y en línea, en el año 2021, representando un hallazgo muy relevante para este estudio (Larios et al., 2021).

Una muestra de lo aseverado en el párrafo anterior, constituye el hecho de que una empresa colombiana que expende diferentes comidas rápidas y otros alimentos en restaurantes, de las marcas Dominó Pizzas, Starbucks, Archies y otros, implementaron una alianza estratégica para la implantación del servicio a domicilio, como una opción para favorecer el incremento progresivo de las ventas, durante la cuarentena de los meses de marzo, abril y mayo en el vecino país de Colombia (Hernández, 2020). Se puede añadir a ello, lo manifestado por Contreras y Velásquez (2020), quienes han indicado que "solo las empresas que adopten la reinvención, podrán ser competitivas en el futuro inmediato" (p. 15), criterio que ha sido compartido por Montejo, Torres y Quijano (2020), quienes también recomendaron "el servicio a domicilio como una de las opciones para superar la 
crisis económica" (p. 550), que está dejando como consecuencia la infección por coronavirus, a su paso por Latinoamérica y el mundo entero.

Se tomó también otro estudio realizado en Colombia, el cual expone que es necesario replantear el modelo de negocios existente actualmente en la microempresa, donde la tecnología y el servicio a domicilio constituyen herramientas que puedan aportar en gran medida a la permanencia de los negocios y a su fortalecimiento en tiempos de pandemia (Matallana, 2020).

Mientras tanto, San Vicente (2020) expone "el papel activo de la bicicleta en tiempos de COVID - 19, para satisfacer necesidades de mensajería, transporte e inclusive el servicio a domicilio, en tiempos donde se restringe la circulación vehicular y hasta la movilidad de personas" (p. 3, 4), en horarios establecidos previamente por los diferentes confinamientos que tienen lugar en las diferentes naciones latinoamericanas y del mundo entero, pero que flexibiliza la entrega de productos a domicilio y el trabajo en los sectores estratégicos.

De este modo, resulta urgente el cambio de modelo económico para superar la crisis del COVID - 19 (Rojo y Bonilla, 2020). Así está tomado mayor forma el servicio a domicilio, como un mecanismo de utilidad práctica para que los microempresarios puedan permanecer con sus negocios, en un mercado que se ha visto severamente deteriorado por las restricciones en 14 meses de pandemia.

En definitiva, los principales beneficiarios del servicio a domicilio y el comercio electrónico, son las MIPYMES, quienes pueden impulsar su oferta, permanecer en el negocio, mantener su fuerza laboral y el empleo pleno, para superar el desempleo, además de permitir la satisfacción máxima de los clientes, que reciben los productos de su predilección, para colmar sus necesidades y expectativas (Rodríguez et al., 2020).

Con este antecedente, se destaca como objetivo principal del presente artículo, establecer la importancia del servicio a domicilio en la permanencia de los negocios en tiempos de la pandemia COVID - 19, del cual se derivan como objetivos específicos: identificar la variación porcentual de las ventas de las microempresas, antes, durante y después de los confinamientos del año 2020 y 2021, así como, determinar la relación existente entre las variaciones de ingresos obtenidas por las microempresas, en los años 2020 y 2021, para luego indicar si el servicio a domicilio tuvo o no una influencia en las ventas de las empresas y si constituye o no una herramienta útil para el logro de la permanencia de estos pequeños negocios, considerando para el efecto, una muestra de establecimientos en el sur de la ciudad de Guayaquil.

\section{Metodología}

El presente artículo aplicó la metodología descriptiva, con enfoque cuantitativo, debido a que expone en cifras numéricas y porcentuales, las variaciones de los ingresos obtenidos por una muestra de microempresas del sur de Guayaquil (Valdiviezo y Jaramillo, 2019), detallando la evolución de las variables y su significado (Monjarás et al., 2017), para 
establecer una correlación entre estas cifras y cumplir con los objetivos de la investigación.

Mientras tanto, la población seleccionada se ubicó en los establecimientos comerciales de la ciudad de Guayaquil, que según el censo económico realizado por el (INEC, 2020), asciende a 58.290 comercios. Debido al elevado número de locales, para la obtención de la muestra, se aplicó el muestreo probabilístico con el criterio de universo desconocido (Lúa y Zambrano, 2019), por ser el mismo superior a 10.000 elementos, a través de la siguiente ecuación estadística:

$$
\mathrm{n}=\frac{\mathrm{Z}^{2} \times \mathrm{p} \times \mathrm{q}}{\mathrm{d}^{2}}
$$

Simbología:

- $\mathrm{n}=$ Tamaño de muestra

- $\mathrm{p}=$ probabilidad de éxito $=0,5$

- $\mathrm{q}=$ probabilidad de fracaso $=0,5$

- $\mathrm{d}^{2}=$ error de la medición $(5 \%)$.

- $\mathrm{Z}^{2}=$ Coeficiente de corrección del error $(1,96)$.

$$
\mathrm{n}=\frac{(1,96)^{2} \times(0,5) \times(0,5)}{(5 \%)^{2}}=384 \text { encuestas }
$$

La muestra fue igual a 384 establecimientos comerciales del sur de Guayaquil, a cuyos propietarios o representantes, se formuló el instrumento investigativo correspondiente. En efecto, la técnica de investigación aplicada en este artículo, fue la encuesta, la cual constó del instrumento del cuestionario con preguntas cerradas, formulada a propietarios o representantes de 384 establecimientos del sur de Guayaquil, tomados como muestra poblacional.

Por otra parte, se aplicó el coeficiente de Pearson para establecer la correlación entre las variaciones de los ingresos por ventas, antes, durante y después de los confinamientos del 2020 y 2021, en Guayaquil. Con esta metodología, se ha podido indicar si el servicio a domicilio tuvo o no una influencia en las ventas de las empresas y si constituye o no una herramienta útil para el logro de la permanencia de estos pequeños negocios.

\section{Resultados}

Los resultados de la investigación de campo, se han interpretado de acuerdo a los siguientes gráficos obtenidos con la aplicación de las funciones del programa Microsoft Excel: 


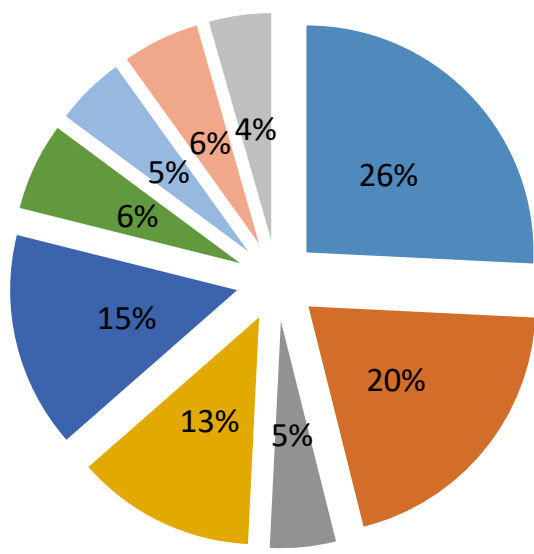

- Tienda

Restaurante

- Heladería

- Panadería

- Venta de comida rápida

Bazar

Novedades

Tercena

Figura 1. Tipo de negocio.

Fuente: Encuestas aplicadas por los autores a los propietarios o representantes de establecimientos comerciales.

Se desprende de los resultados obtenidos sobre el tipo de negocio, que las tiendas representan 26\% de los establecimientos comerciales de la muestra seleccionada, siguiéndole en orden de importancia, los restaurantes (20\%), las ventas de comida rápida $(15 \%)$ y las panaderías (13\%). El resto de establecimientos conforman alrededor de la cuarta parte $(26 \%)$ de la muestra escogida.

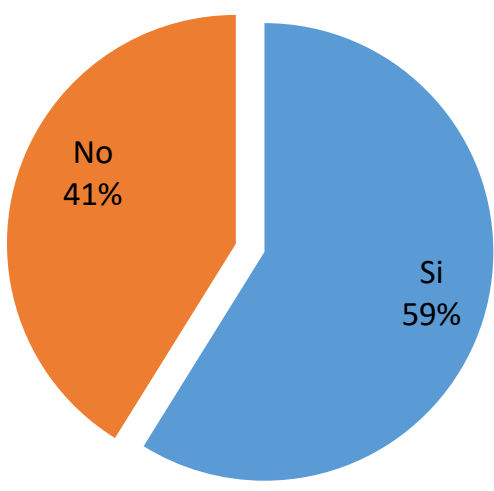

Figura 2. Aplicó el servicio a domicilio en el confinamiento del 2020.

Fuente: Encuestas aplicadas por los autores a los propietarios o representantes de establecimientos comerciales.

De los resultados obtenidos, se pone de manifiesto que el 59\% de los negocios microempresariales, si optó por el servicio a domicilio durante el confinamiento del año 2020, a diferencia del $41 \%$ que no se benefició de este mecanismo para vender en el tiempo que duró la cuarentena, durante los meses de marzo, abril y mayo del 2020. 


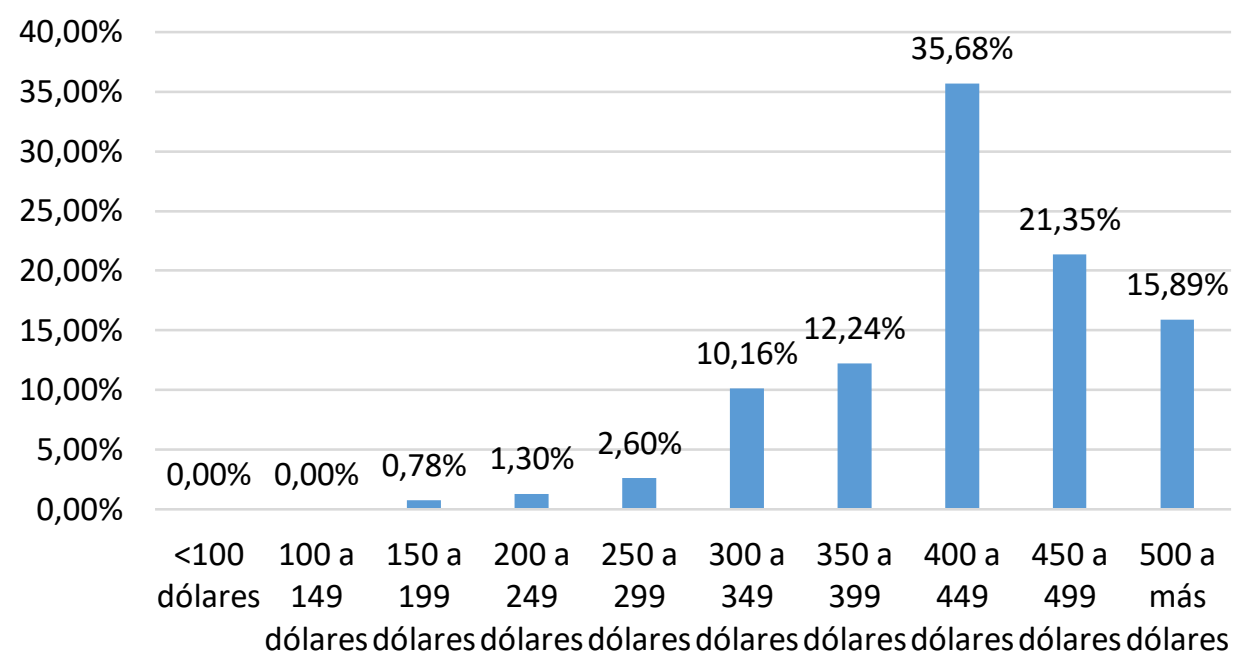

Figura 3. Ingresos semanales promedios antes del confinamiento del 2020.

Fuente: Encuestas aplicadas por los autores a los propietarios o representantes de establecimientos comerciales.

Se pudo conocer, que antes del confinamiento que inició el mes de marzo del 2020, el $35,68 \%$ de los establecimientos comerciales, tenía ingresos por ventas, entre $\$ 400,00$ a $\$ 499,00$ semanales, mientras que los ingresos del $21,35 \%$ de negocios microempresariales, oscilaban entre $\$ 450,00$ a $\$ 499,00$ por semana, pero hubo un $15,89 \%$ de locales que vendían más de $\$ 500,00$ en este mismo periodo de tiempo. Asimismo, $12,24 \%$ de negocios vendían alrededor de $\$ 350,00$ a $\$ 399,00, y, 10,16 \%$ de establecimientos comerciales ingresaron entre $\$ 300,00$ a $\$ 349,00$ por semana. En cambio, menos del 5\% de comercios, vendían menos de \$300,00 semanales.

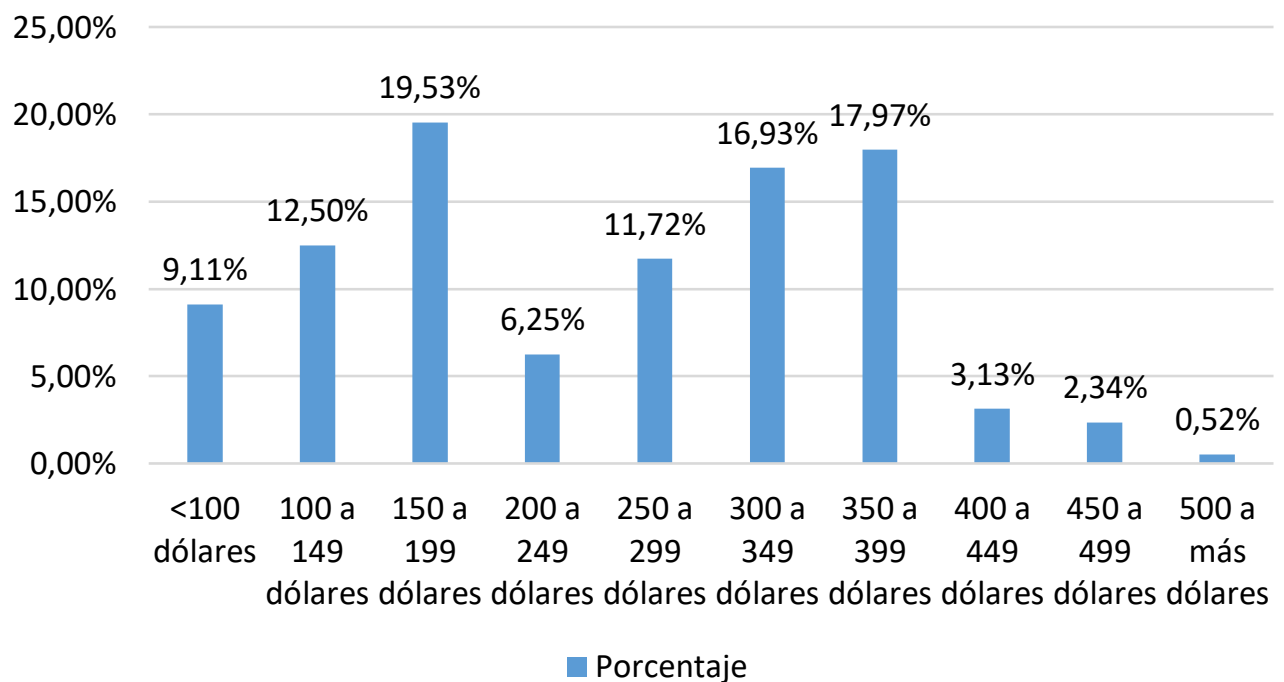

Figura 4. Ingresos semanales promedios durante el confinamiento del 2020.

Fuente: Encuestas aplicadas por los autores a los propietarios o representantes de establecimientos comerciales.

Se pudo conocer, que durante el confinamiento que inició el mes de marzo del 2020, el $17,97 \%$ de los establecimientos comerciales, tenía ingresos por ventas, entre $\$ 350,00$ a 
$\$ 399,00$ semanales, mientras que los ingresos del $16,93 \%$ de negocios microempresariales, oscilaban entre $\$ 300,00$ a $\$ 349,00$ por semana, pero hubo un $12,50 \%$ de locales que solo vendió $\$ 100,00$ a $\$ 149,00$ semanales, así como un $19,53 \%$ de comercios microempresariales, que solo vendió de $\$ 150,00$ a $\$ 199,00$ en este mismo periodo de tiempo. Asimismo, $11,72 \%$ de negocios vendían alrededor de $\$ 250,00$ a $\$ 299,00$, entre tanto que $9,11 \%$ de establecimientos comerciales ingresaron menos de $\$ 100,00$ por semana y $6,25 \%$ vendieron de $\$ 200,00$ a $\$ 249,00$ semanales. En cambio, menos del $6 \%$ de comercios, vendieron más de $\$ 400,00$ semanales, existiendo un gran contraste con lo acontecido antes de este confinamiento.

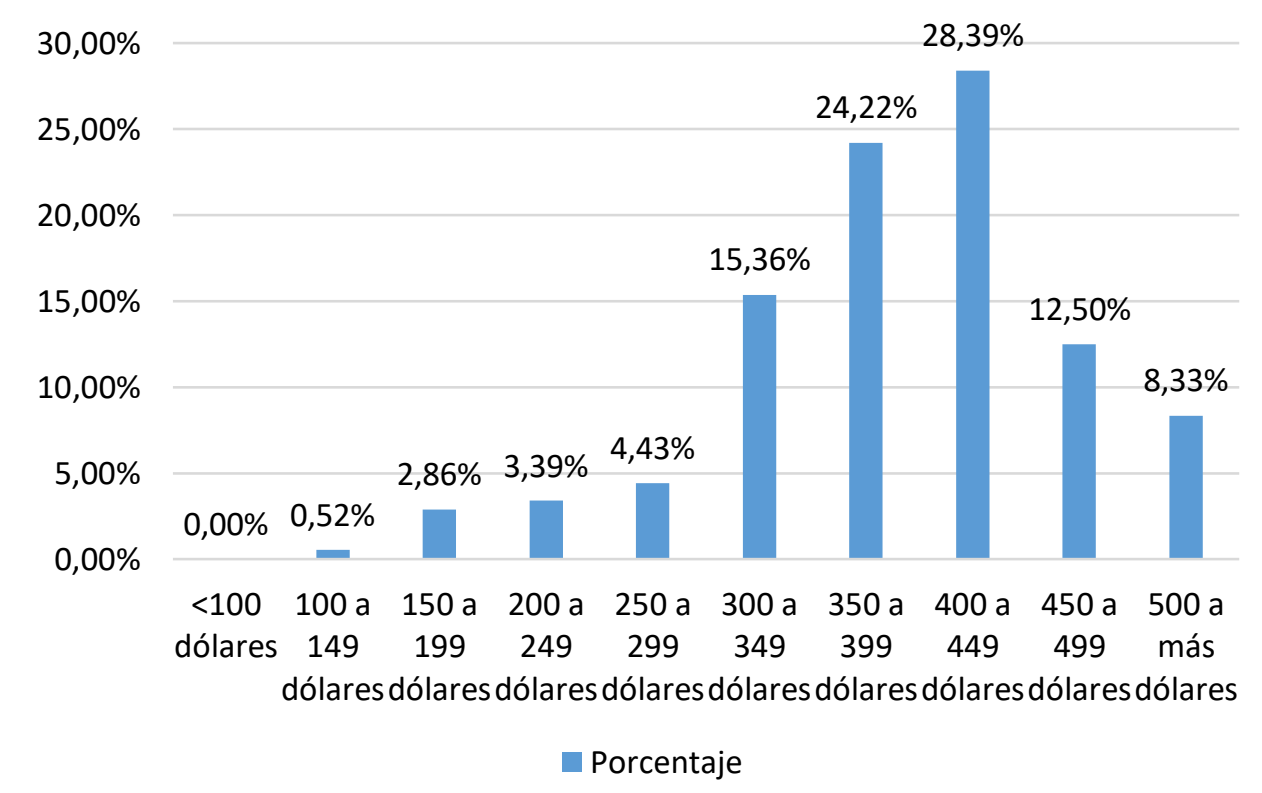

Figura 5. Ingresos semanales promedios después del confinamiento del 2020.

Fuente: Encuestas aplicadas por los autores a los propietarios o representantes de establecimientos comerciales.

Se pudo conocer, que después del confinamiento que inició el mes de marzo del 2020, el $28,39 \%$ de los establecimientos comerciales, tenía ingresos por ventas, entre $\$ 400,00$ a $\$ 499,00$ semanales, mientras que los ingresos del $24,22 \%$ de negocios microempresariales, oscilaban entre $\$ 350,00$ a $\$ 399,00$ por semana, pero hubo un $15,36 \%$ de locales que vendían entre $\$ 300,00$ a $\$ 349,00$ en este mismo periodo de tiempo. Asimismo, solo $8,33 \%$ de negocios vendieron más de $\$ 500,00$, y, algo más del $10 \%$ de establecimientos comerciales ingresaron menos de $\$ 300,00$ semanales, es decir, disminuyeron en algo las ventas de los comercios microempresariales, posterior al confinamiento de marzo, abril y mayo del 2020, en comparación con el promedio de ingresos de los meses anteriores a esta cuarentena. 


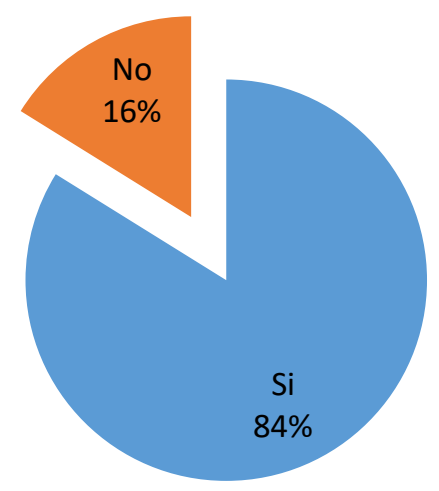

Figura 6. Aplicó el servicio a domicilio en el confinamiento del 2021.

Fuente: Encuestas aplicadas por los autores a los propietarios o representantes de establecimientos comerciales.

De los resultados obtenidos, se pone de manifiesto que el $84 \%$ de los negocios microempresariales, si optó por el servicio a domicilio durante el confinamiento del año 2020, a diferencia del 16\% que no se benefició de este mecanismo para vender en el tiempo que duró la cuarentena, durante los meses de abril y mayo del 2021.

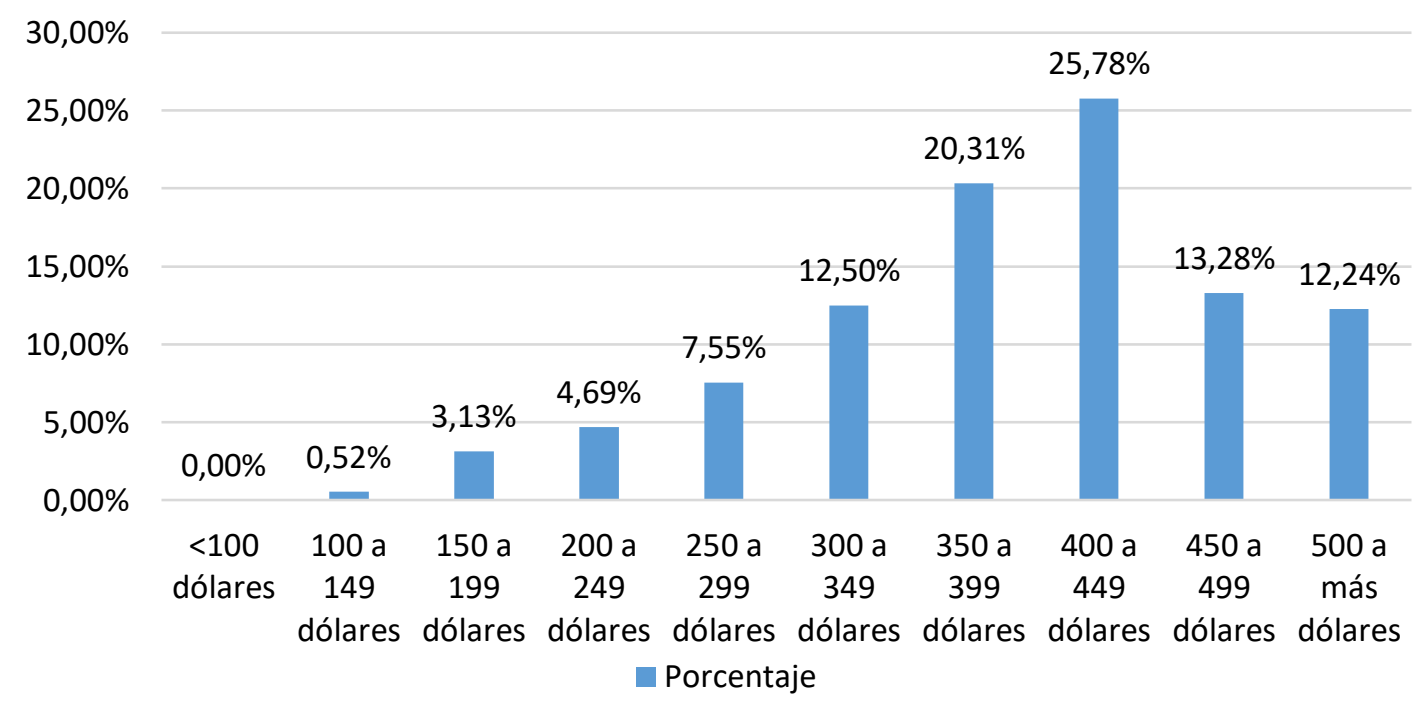

Figura 7. Ingresos semanales promedios antes del confinamiento del 2021.

Fuente: Encuestas aplicadas por los autores a los propietarios o representantes de establecimientos comerciales.

Se pudo conocer, que antes del confinamiento que inició el mes de abril del 2021, el $25,78 \%$ de los establecimientos comerciales, tenía ingresos por ventas, entre $\$ 400,00$ a $\$ 449,00$ semanales, mientras que los ingresos del $20,31 \%$ de negocios microempresariales, oscilaban entre $\$ 350,00$ a $\$ 399,00$ por semana, $y, 12,50 \%$ de establecimientos vedieron entre $\$ 300,00$ a $\$ 349,00$ semanales. Pero hubo un $12,24 \%$ de locales que vendían más de $\$ 500,00$ y un $13,28 \%$ que obtuvieron ingresos entre $\$ 450,00$ a $\$ 499,00$, en este mismo periodo de tiempo. En cambio, el 15,89\% de comercios, vendían menos de $\$ 300,00$ semanales, es decir, el triple de los establecimientos que vendían menos de esa cifra de dólares por semana, previo al confinamiento del 2020. 


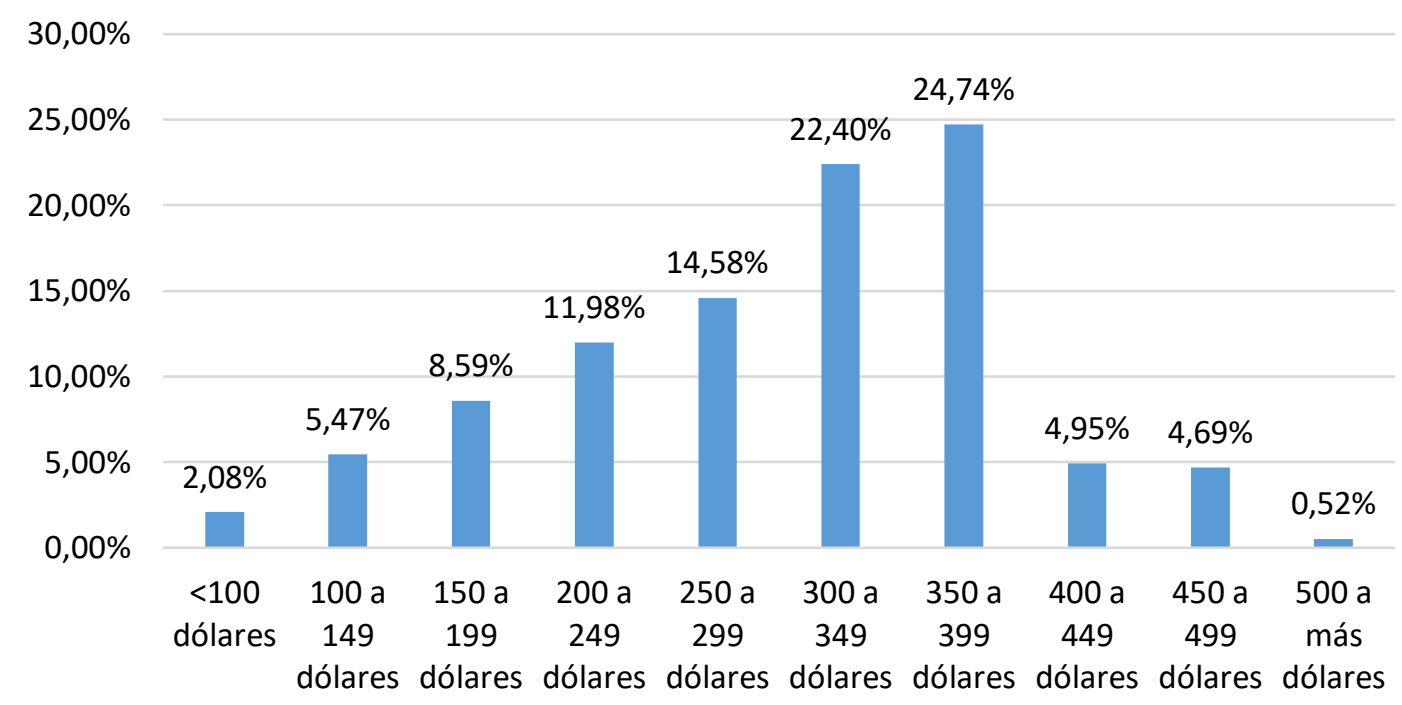

Figura 8. Ingresos semanales promedios durante el confinamiento del 2021.

Fuente: Encuestas aplicadas por los autores a los propietarios o representantes de establecimientos comerciales.

Se pudo conocer, que durante el confinamiento que inició el mes de abril del 2021, el $24,74 \%$ de los establecimientos comerciales, tenía ingresos por ventas, entre $\$ 350,00$ a $\$ 399,00$ semanales, mientras que los ingresos del $22,40 \%$ de negocios microempresariales, oscilaban entre $\$ 300,00$ a $\$ 349,00$ por semana, pero hubo un $14,58 \%$ de locales que solo vendió \$250,00 a \$299,00 semanales, así como un 11,98\% de comercios microempresariales, que solo vendió de $\$ 200,00$ a $\$ 249,00$ en este mismo periodo de tiempo. Asimismo, el 10\% de negocios superó los $\$ 500,00$ en ventas, mientras que $16 \%$ de negocios vendieron menos de $\$ 200,00$ semanales, encontrándose una mejoría en lo relacionado al confinamiento del 2020, donde los negocios que no optaron por el servicio a domicilio, fueron el triple que en el año 2021.

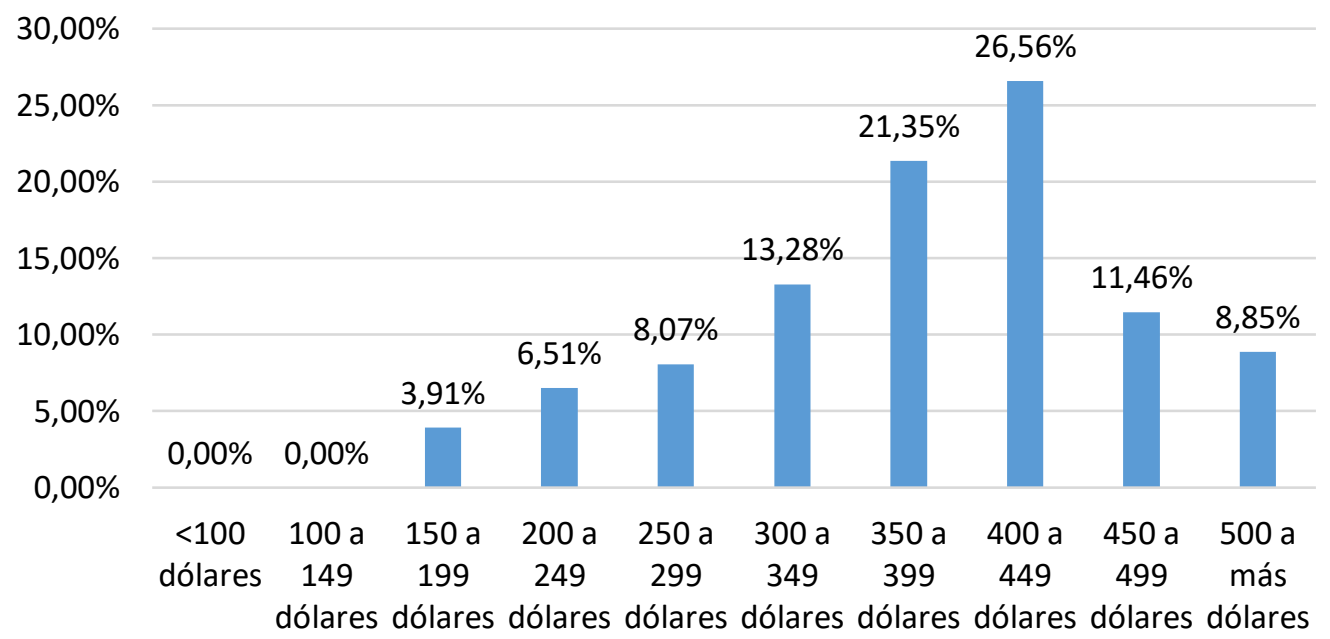

Figura 9. Ingresos semanales promedios después del confinamiento del 2021.

Fuente: Encuestas aplicadas por los autores a los propietarios o representantes de establecimientos comerciales. 
Se pudo conocer, que después del confinamiento que inició el mes de abril del 2021, el $26,56 \%$ de los establecimientos comerciales, tenía ingresos por ventas, entre $\$ 400,00$ a $\$ 499,00$ semanales, mientras que los ingresos del $21,35 \%$ de negocios microempresariales, oscilaban entre $\$ 350,00$ a $\$ 399,00$ por semana, pero hubo un $13,28 \%$ de locales que vendían entre $\$ 300,00$ a $\$ 349,00$ en este mismo periodo de tiempo. Asimismo, solo 8,85\% de negocios vendieron más de \$500,00, y, algo más del $20 \%$ de establecimientos comerciales ingresaron menos de $\$ 300,00$ semanales, es decir, que los ingresos de los comercios microempresariales, se mantuvieron casi a la par, con una disminución mínima, posterior al confinamiento de abril y mayo del 2021, en comparación con el promedio de ingresos de los meses del 2021, anteriores a esta cuarentena, evidenciándose los beneficios del servicio a domicilio en la muestra seleccionada.

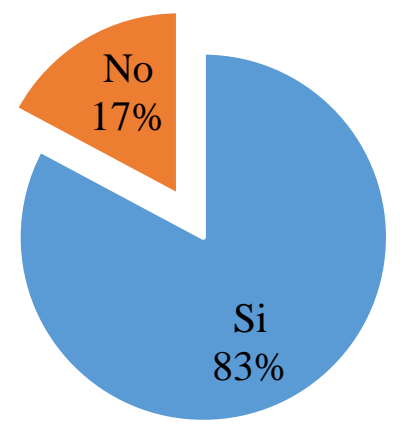

Figura 10. Servicio a domicilio solventó costos fijos.

Fuente: Encuestas aplicadas por los autores a los propietarios o representantes de establecimientos comerciales.

De los resultados obtenidos, se pone de manifiesto que el $83 \%$ de los negocios microempresariales, si pudo solventar los gastos por alquiler, con la incorporación del servicio a domicilio durante los confinamientos de los años 2020 y 2021, a diferencia del $17 \%$ que manifestó lo contrario y no pudo solventar estos gastos.

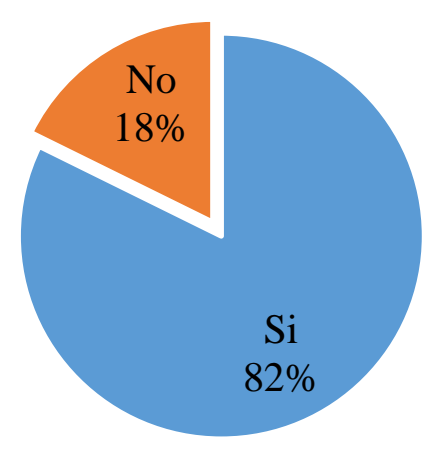

Figura 11. Servicio a domicilio solventó pago de sueldos del personal.

Fuente: Encuestas aplicadas por los autores a los propietarios o representantes de establecimientos comerciales. 
De los resultados obtenidos, se pone de manifiesto que el $82 \%$ de los negocios microempresariales, si pudo solventar los gastos por sueldos del personal, con la incorporación del servicio a domicilio durante los confinamientos de los años 2020 y 2021, a diferencia del $18 \%$ que manifestó lo contrario y no pudo solventar estos gastos.

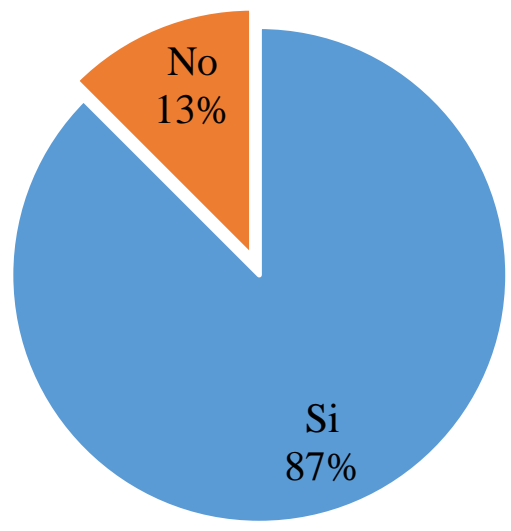

Figura 12. Servicio a domicilio favoreció la permanencia del negocio.

Fuente: Encuestas aplicadas por los autores a los propietarios o representantes de establecimientos comerciales.

De los resultados obtenidos, se pone de manifiesto que el $87 \%$ de los propietarios y/o administradores de los establecimientos microempresariales, indicaron que el servicio a domicilio favoreció la permanencia de su negocio, a diferencia del 13\% que manifestó lo contrario sobre este particular.

\begin{tabular}{lcrrrrr}
\hline & \multicolumn{3}{c}{ Confinamiento 2020 } & \multicolumn{3}{c}{ Confinamiento 2021 } \\
\multicolumn{1}{c}{ Detalle } & Antes & \multicolumn{1}{c}{ Durante } & \multicolumn{1}{c}{ Después } & \multicolumn{1}{c}{ Antes } & \multicolumn{1}{c}{ Durante } & \multicolumn{1}{c}{ Después } \\
\hline$<100$ dólares & 0,00 & $2.625,00$ & 0,00 & 0,00 & 600,00 & 0,00 \\
100 a 149 dólares & 0,00 & $6.000,00$ & 250,00 & 250,00 & $2.625,00$ & 0,00 \\
150 a 199 dólares & 525,00 & $13.125,00$ & $1.925,00$ & $2.100,00$ & $5.775,00$ & $2.625,00$ \\
200 a 249 dólares & $1.125,00$ & $5.400,00$ & $2.925,00$ & $4.050,00$ & $10.350,00$ & $5.625,00$ \\
250 a 299 dólares & $2.750,00$ & $12.375,00$ & $4.675,00$ & $7.975,00$ & $15.400,00$ & $8.525,00$ \\
300 a 349 dólares & $12.675,00$ & $21.125,00$ & $19.175,00$ & $15.600,00$ & $27.950,00$ & $16.575,00$ \\
350 a 399 dólares & $17.625,00$ & $25.875,00$ & $34.875,00$ & $29.250,00$ & $35.625,00$ & $30.750,00$ \\
400 a 449 dólares & $58.225,00$ & $5.100,00$ & $46.325,00$ & $42.075,00$ & $8.075,00$ & $43.350,00$ \\
450 a 499 dólares & $38.950,00$ & $4.275,00$ & $22.800,00$ & $24.225,00$ & $8.550,00$ & $20.900,00$ \\
500 a más dólares & $32.025,00$ & $1.050,00$ & $16.800,00$ & $24.675,00$ & $1.050,00$ & $17.850,00$ \\
Promedio & 426,82 & 252,47 & 389,97 & 391,15 & 302,08 & 380,73 \\
& & $-40,85 \%$ & $-8,63 \%$ & & $-22,77 \%$ & $-2,66 \%$ \\
\hline
\end{tabular}

Tabla 1. Detalle de ingresos antes, durante y después de los confinamientos del 2020 y 2021.

Fuente: Encuestas aplicada a propietarios o representantes de establecimientos comerciales.

Con base en los resultados obtenidos, se pudo establecer los promedios de ingresos por ventas, antes, durante y después del confinamiento del año 2020, observándose una reducción de las ventas del 40,85\% durante la cuarentena de los meses de marzo, abril y 
mayo del 2020. Sin embargo, después del confinamiento en mención, las ventas volvieron a incrementarse, pero no a los niveles que mantuvieron antes de la cuarentena, evidenciándose una reducción del 8,63\% de las ventas en estos negocios, al comparar el antes y del después del confinamiento del 2020.

Prosiguiendo con el análisis de los resultados obtenidos, se pudo establecer los promedios de ingresos por ventas, antes, durante y después del confinamiento del año 2021, observándose una reducción de las ventas del 22,77\% durante la cuarentena de los meses de abril y mayo del 2020. Sin embargo, después del confinamiento en mención, las ventas volvieron a incrementarse, casi a los niveles que mantuvieron antes de la cuarentena del mismo periodo anual, evidenciándose tan sólo una reducción del 2,66\% de las ventas en estos negocios, al comparar el antes y del después del confinamiento del 2021.

Posterior a esta interpretación, se ejecutó el procedimiento con las funciones del programa Excel, para determinar el coeficiente de Pearson de las curvas de ingresos, antes, durante y después de los confinamientos del 2020 y 2021, considerando las siguientes restricciones:

- Si el coeficiente Pearson > 0,9: servicio a domicilio tiene correlación con la variación de las ventas de los establecimientos comerciales; caso contrario; si coeficiente Pearson < 0,9: servicio a domicilio no tiene correlación con la variación de las ventas de los establecimientos comerciales.

- Si el p valor < 0,05: servicio a domicilio tiene correlación con la variación de las ventas de los establecimientos comerciales; caso contrario; si coeficiente p valor $>$ 0,05: servicio a domicilio no tiene correlación con la variación de las ventas de los establecimientos comerciales.

Al operar las funciones del programa Microsoft Excel, mediante las cuales se puede realizar el cálculo del coeficiente de Pearson, se observaron los siguientes resultados:

\begin{tabular}{lrr}
\hline Confinamiento 2020 y 2021 & Pearson & p value \\
\hline Antes & 0,9270618 & 0,0341426 \\
Durante & 0,924352 & 0,0342533 \\
Después & 0,9929333 & 0,0070667 \\
\hline
\end{tabular}

Tabla 2. Detalle de coeficiente de Pearson de curvas de ingresos antes, durante y después de los confinamientos del 2020 y 2021.

Fuente: Encuestas aplicada a propietarios o representantes de establecimientos comerciales.

El coeficiente de Pearson señaló un parámetro de 0,9271 antes de los confinamientos, con un p valor de 0,0341 , además de un coeficiente de 0,9244 durante las cuarentenas, con un $\mathrm{p}$ valor de 0,0343, y, un coeficiente de 0,9929 después de los confinamientos, con un $\mathrm{p}$ valor de 0,0071. Se observa que todos los coeficientes de Pearson son superiores a 0,90 y que los $\mathrm{p}$ valor son menores a 0,05, el máximo error admisible en la medición estadística, por consiguiente, se establece que existe correlación entre el servicio a domicilio y las variaciones de las ventas, previo, durante y después de los confinamientos de los años 2020 y 2021. 


\section{Conclusiones}

- Se concluye, en primer lugar, que las variaciones de las ventas semanales de los establecimientos microempresariales fue a la baja durante los confinamientos del 2020 y 2021, en comparación con los que se vendía antes de las cuarentenas, recuperándose posterior a los confinamientos, aunque no en el mismo nivel que tenían previamente. Sin embargo, se pudo conocer que un $100 \%$ más de establecimientos económicos que optaron por la estrategia del servicio a domicilio, obtuvieron mayores ingresos por ventas durante el confinamiento del 2021, en comparación con la cuarentena del año 2020, reduciéndose a la mitad los negocios que vendieron menos de \$200,00 durante la cuarentena del 2021, en comparación con la del 2020.

- De este modo, en segundo lugar, se observó una correlación directa, con coeficientes de Pearson superiores a 0,9 y p valores menores a 0,05, entre las variaciones de los ingresos obtenidos por los negocios micro empresariales, durante los confinamientos de los años 2020 y 2021, observándose que mientras una mayor cantidad de negocios aplicaron el servicio a domicilio, sus ventas se incrementaron y sobretodo, pudieron permanecer en el mercado, con ingresos algo sustentables, superiores a $\$ 300,00$, posterior a los confinamientos.

- En consecuencia, los ingresos por ventas de los negocios microempresariales se incrementaron $20 \%$ en el confinamiento del 2021, en comparación con las ventas durante la cuarentena del 2020, estabilizándose también los ingresos de estos negocios pequeños, posterior al confinamiento, a fines de mayo del 2021, situación que indica con claridad la importancia del servicio a domicilio, como mecanismo que ha favorecido la permanencia de los negocios en tiempos de la pandemia COVID - 19.

- Se recomienda ante esta situación, que los propietarios y administradores de las microempresas que trabajan en la ciudad de Guayaquil, adoptar el servicio a domicilio y los dispositivos tecnológicos (TIC), como herramientas que pueden contribuir al desarrollo de las actividades económicas, durante la pandemia y que puede tener un impacto positivo relevante, en el tiempo postpandemia.

\section{Referencias Bibliográficas}

Barahona, R. (2021). Análisis del comportamiento de los precios al consumidor previo y posterior al COVID19 en el Ecuador. Guayaquil: Universidad Espíritu Santo.

Blackman, A., Ibáñez, A., Izquierdo, A., Keefer, P., Moreira, M., Schady, N., \& Serebrisky, T. (2020). La Política pública frente al Covid-19: Recomendaciones para América Latina y el Caribe. Washington: Banco Interamericano de Desarrollo.

Canseco , R., Vásquez, E., Valcárcel, L., Torres, L., Butler, M., Canales, C., . . Koechlin, J. (2020). Reinventando el turismo en tiempos de covid-19. Lima: Universidad 
San Ignacio de $\quad$ Loyola $\quad$ Obtenido de http://repositorio.usil.edu.pe/bitstream/USIL/9891/1/2020_DiezCanseco_Reinventando-el-turismo.pdf

Casco, A. (2020). Efectos de la pandemia de COVID-19 en el comportamiento del consumidor. Innovare, $9(2), \quad 19 . \quad$ Obtenido de https://camjol.info/index.php/INNOVARE/article/view/10208

Castro, S. (2020). Comportamiento del mercado local y estrategias de ventas locales frente a la pandemia por Covid-19 en la ciudad de Manta. REICOMUNICAR, 3(6), 16. Obtenido de http://reicomunicar.org/index.php/reicomunicar/article/view/3

Contreras, R., \& Velásquez, N. (2020). Los Efectos de la Pandemia: El Coronavirus en Colombia. Bogotá: Universidad Católica de Colombia.

Coronel, C., Ignacio, H., Quispe, L., \& Rivera, E. (2020). Ecosistema inteligente para bodegas en el contexto de la COVID-19. Lima: PUCP.

Del Pino , T., \& Li Gayoso, H. (2020). Adecuación del servicio e infraestructura de restaurantes ante la COVID-19 y su aplicación en el Perú. Lima: Repositorio académico UPC. Obtenido de https://repositorioacademico.upc.edu.pe/handle/10757/654051

Giles, C. (2020). Recomendaciones para las MIPyME ¿Qué hacer para sobrevivir a la pandemia del Covid-19? Notas Estratégicas, 86(06), 16. Obtenido de http://www.bibliodigitalibd.senado.gob.mx/bitstream/handle/123456789/4845/N ota86_RecomendacionesMipymePdf.pdf?sequence=8\&isAllowed=y

Hernández, J. (2020). Estrategias comerciales de alianzas para la potencialización y aumento de ventas durante la cuarentena del COVID-19 en Alsea Colombia. Bogotá: Universidad Santo Tomás. Obtenido de https://repository.usta.edu.co/handle/11634/28010

INEC. (2020). Censo económico. Quito: INEC.

INEC. (2020). Índice de precios al consumidor 2019. Ecuador: Boletín técnico $\mathrm{N}^{\circ} 10$ 2020-IPC.

Larios, E., Fischer , L., \& Monteiro, T. (2021). Comportamiento de compra generacional en época de covid-19: Un estudio transversal en México, Brasil, Colombia y Ecuador. Inclusiones, $8(07), \quad 16 . \quad$ Obtenido de http://revistainclusiones.org/index.php/inclu/article/view/1100

Lúa, A., \& Zambrano, J. (2019). Plan Estratégico Para Mejorar El Posicionamiento Y Rentabilidad Del Taller Mecánico Zambrano De La Ciudad De Guayaquil. Guayaquil: Universidad Laica Vicente Rocafuerte. 
Matallana, L. (2020). Retos Empresariales Tras el Coronavirus COVID 19. Bogotá: Universidad Católica de Colombia. Obtenido de https://repository.ucatolica.edu.co/bitstream/10983/24668/1/Ensayo\%20final.pdf

Ministerio de Salud Pública. (2020). Acuerdo Ministerial No. 00126-2020. Quito: MSP.

Monjarás, A., Bazán, A., Pacheco, Z., Rivera, J., Zamarripa, J., \& Cuevas, C. (2017). Diseños de investigación. Educación y Salud, 8(15), 15.

Montejo, M., Torres, M., \& Quijano, S. (2020). Adaptación al cambio en tiempo de contingencia sanitaria covid-19 en el sector comercial. México: UniversidadInstituto Tecnológico Superior de Escárcega.

Palomino , P., \& Palomino, A. (2021). Análisis del efecto del valor capital de marca en el comportamiento del consumidor en el contexto de crisis sanitaria a causa de la COVID-19: Caso de estudio LA BADIANE. Lima: PUCP.

Presidencia de la República del Ecuador. (2020). Decreto Ejecutivo No. 1052. Quito: Presidencia de la República del Ecuador.

Presidencia de la República del Ecuador. (2020). Decreto Ejecutvio No. 1017. Quito: Presidencia de la República del Ecuador.

Rodríguez, K., Ortiz, O., Quiroz, A., \& Parrales, M. (2020). El e-commerce y las Mipymes en tiempos de Covid-19. Espacios, 41(9), 17.

Rojo, M., \& Bonilla, D. (2020). COVID-19: La necesidad de un cambio de paradigma económico y social. CienciAmérica, 9(2), 17.

San Vicente, L. (2020). El papel de la bicicleta en tiempos de COVID-19: pedalear para transformar. Jalisco: Plataforma de Conocimiento para la Transformación Urbana.

Valdiviezo, X., \& Jaramillo, M. (2019). Metodología de investigación cuantitativa en trabajos de graduación de la modalidad de titulación de la carrera de contabilidad y auditaría. Machala: Universidad Técnica de Machala.

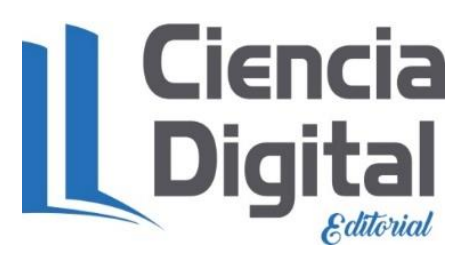




\section{PARA CITAR EL ARTÍCULO INDEXADO.}

Estéfano Almeida, M. A., Llerena Erazo, E. B., Morales González, P. B., \& Quiroz Moncerrad, J. F. (2021). Estrategia del servicio a domicilio y permanencia de negocios en tiempos de COVID-19. AlfaPublicaciones, 3(3), 23-39. https://doi.org/10.33262/ap.v3i3.65

\section{\Ciencia}

El artículo que se publica es de exclusiva responsabilidad de los autores y no necesariamente reflejan el pensamiento de la Revista Alfa Publicaciones.

El artículo queda en propiedad de la revista y, por tanto, su publicación parcial y/o total en otro medio tiene que ser autorizado por el director de la Revista Alfa Publicaciones.
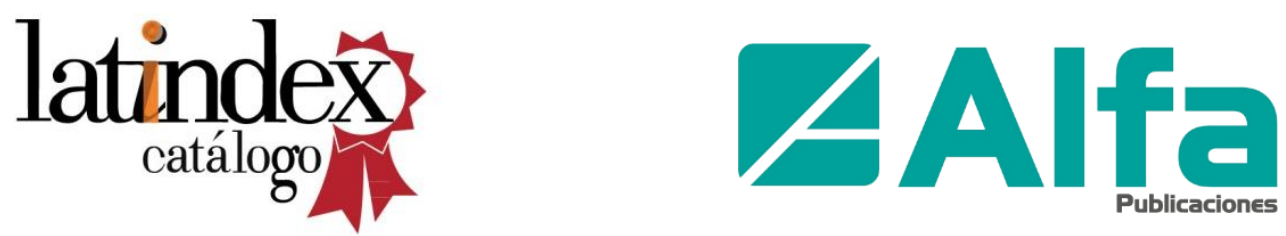